\title{
Fox Chase Cancer Center
}

\author{
Robert C. Young, M.D., President
}

Fox Chase Cancer Center, one of 26 National Cancer Institute-designated comprehensive cancer centers in the nation, is dedicated to the mission of reducing the burden of human cancer. The center's activities include basic, clinical, and prevention research; detection and treatment of cancer; and community outreach programs.

Fox Chase Cancer Center was formed in 1974 by the union of the American Oncologic Hospital and the Institute for Cancer Research. Now known as the Hospital of Fox Chase Cancer Center, the American Oncologic Hospital was the first cancer hospital in the nation. Although cancer was then considered incurable, a group of Philadelphia leaders established the hospital in 1904 to care for patients and to carry out research that would make cancer a manageable disease.

Founded in 1927, the Institute for Cancer Research helped change the course of cancer research, which until then had focused solely on studies of tumor tissues. Institute founder Stanley P. Reimann, M.D., believed that to understand the abnormal growth processes of cancer cells, scientists from many disciplines must study the basic mechanisms of normal cells. This thenrevolutionary approach forms the basis for most cancer research today. With these principles in mind, the Institute grew during the 1950s, '60s, '70s, and '80s, with scientists eager to research new programs such as cell and developmental biology, immunology, virology, genetics and biochemistry.

Today, Fox Chase has more than 1,800 employees and an annual budget of $\$ 127$ million. Research is conducted in more than 75 laboratories by a staff of more than 300 physicians and scientists who hold M.D.s, Ph.D.s, or both. Located in the residential northeast section of Philadelphia and surrounded by a park, Fox Chase's

Address correspondence and reprint requests to: Dr. Robert C. Young, Fox Chase Cancer Center, 7701 Burholme Ave., Philadelphia, PA 19111, U.S.A. Phone: 215-728-2781; fax: 215-728-2571; e-mail: rc_young@fccc.edu environment is conducive to interaction between laboratories and the clinic.

Fox Chase's 100-bed hospital is one of the few facilities in the country devoted entirely to cancer care. More than 130 clinical trials of new prevention, diagnostic, and treatment techniques are under way at any one time. Almost 1,000 new patients a year participate in clinical trials.

Fox Chase is structured around three divisions: medical science, basic science, and population science. Medical science is committed to providing excellent patient care and conducting a broad-based program of clinical research. The division of basic science is renowned worldwide for its work in understanding both normal and abnormal cell growth and development. The goal of the division of population science is to identify people at high risk for cancer and to develop strategies to reduce these risks through programs of prevention and early detection. This structure reflects Fox Chase's belief that collaborations between disciplines translates research into clinical applications much more quickly and effectively.

The center serves as a national resource for translating new research findings into medical applications that may become models for improved cancer detection, treatment, and prevention. Among the notable accomplishments by scientists at Fox Chase was the discovery of the hepatitis B virus by Baruch Blumberg, who received the 1976 Nobel Prize in Medicine for his work. His Fox Chase laboratory also subsequently helped develop the hepatitis B vaccinethe first vaccine capable of preventing a human cancer, since hepatitis B can lead to primary liver cancer.

\section{CELLULAR AND DEVELOPMENTAL BIOLOGY}

Fox Chase scientists Robert Briggs and Thomas King's experiments in nuclear transplantation, using embryonic frog cells, resulted in the first vertebrate "clone" in 1952. 
The first mosaic mammal (a mouse with genes from four parents), developed by Beatrice Mintz at Fox Chase in 1962, provided new ways of studying genetic expression, birth defects, and developmental disorders. Using similar techniques, Fox Chase scientists produced a normal mouse by using genetic material from teratocarcinoma cells, thus showing that this cancer does not involve irreversible genetic change.

For more than 30 years, Robert Perry's work on the organization and expression of ribosomal protein genes, their transcriptional regulation, and translational control has contributed toward understanding of the role of proteins in the cell. Perry and his colleagues at Fox Chase have identified specific segments of DNA that play key roles in gene expression.

\section{BIOCHEMISTRY}

Irwin Rose has worked on the protein ubiquitin found in cells and responsible for targeting other protein molecules for disposal. Rose discovered that ubiquitin helps regulate the important proteins that control cell reproduction. When the apparatus for controlling and processing cell proteins is better understood, scientists will be able to compare its function in normal and cancer cells.

Jenny Glusker has been a leader in crystallography for structural research on proteins and other substances. Her work concentrates on enzymes that let the body convert food to energy, on vitamin $\mathrm{B} 12$, and on cancer-causing agents derived from aromatic hydrocarbons, including components of cigarette smoke. Her laboratory is studying the chemotherapy drug estramustine and chemoprevention drugs such as oltipraz.

\section{IMMUNOBIOLOGY}

Melvin Bosma's 1980 discovery of a Fox Chasebred mouse strain with severe combined immune deficiency (SCID) and subsequent elucidation of the developmental genetic defect provided a model for the corresponding human birth defect and helped scientists study how cells of the immune system develop. Since SCID mice have no natural immunity and cannot reject tissue transplants, they have also become valuable research tools for studying other diseases besides cancer, such as AIDS.

Today, researchers at Fox Chase are exploring the cellular and molecular mechanisms involved in the development and production of antibodies. R. Randy Hardy is working on the development and selection of immunoglobulin $\mathrm{V}$ gene repertoires in subpopulations of B cells.

\section{PHRMACOLOGY AND MEDICAL ONCOLOGY}

Pharmacology and medical oncology research at Fox Chase focuses on developing more effective anticancer drugs and investigating the molecular mechanisms that allow some tumor cells to resist treatment. Many of the center's current studies in these areas focus on breast, ovarian, and prostate cancers.

Fox Chase staff are among the leaders in developing cancer treatments that use biologic response modifiers to help mobilize the immune system against cancer cells. The center conducted the first clinical trials of monoclonal antibodies designed to target gastrointestinal cancer cells for diagnosis and treatment in the early 1980s. This work has led to the development of new hybrid "bi-specific" monoclonal antibodies that may allow physicians to deliver activated immune cells directly to a cancer.

\section{VIROLOGY}

Most viruses that cause cancer in animals are retroviruses, as are the human T-cell leukemia virus and the human immunodeficiency virus that causes AIDS. Several Fox Chase laboratories study retroviruses, which reproduce differently than other viruses.

When virologist Jesse Summers directed the Institute for Cancer Research in the early 1980s, his work focused on the molecular biology of hepatitis B on woodchucks. Today, Anna Marie Skalka, Senior Vice President for Basic Science, is exploring the basic mechanisms of RNA viruses: how they encode and transcribe genetic information, and how they produce and process proteins. This program includes studies of the mechanisms the AIDS virus uses to infect cells. By gaining a better understanding of this retrovirus' 


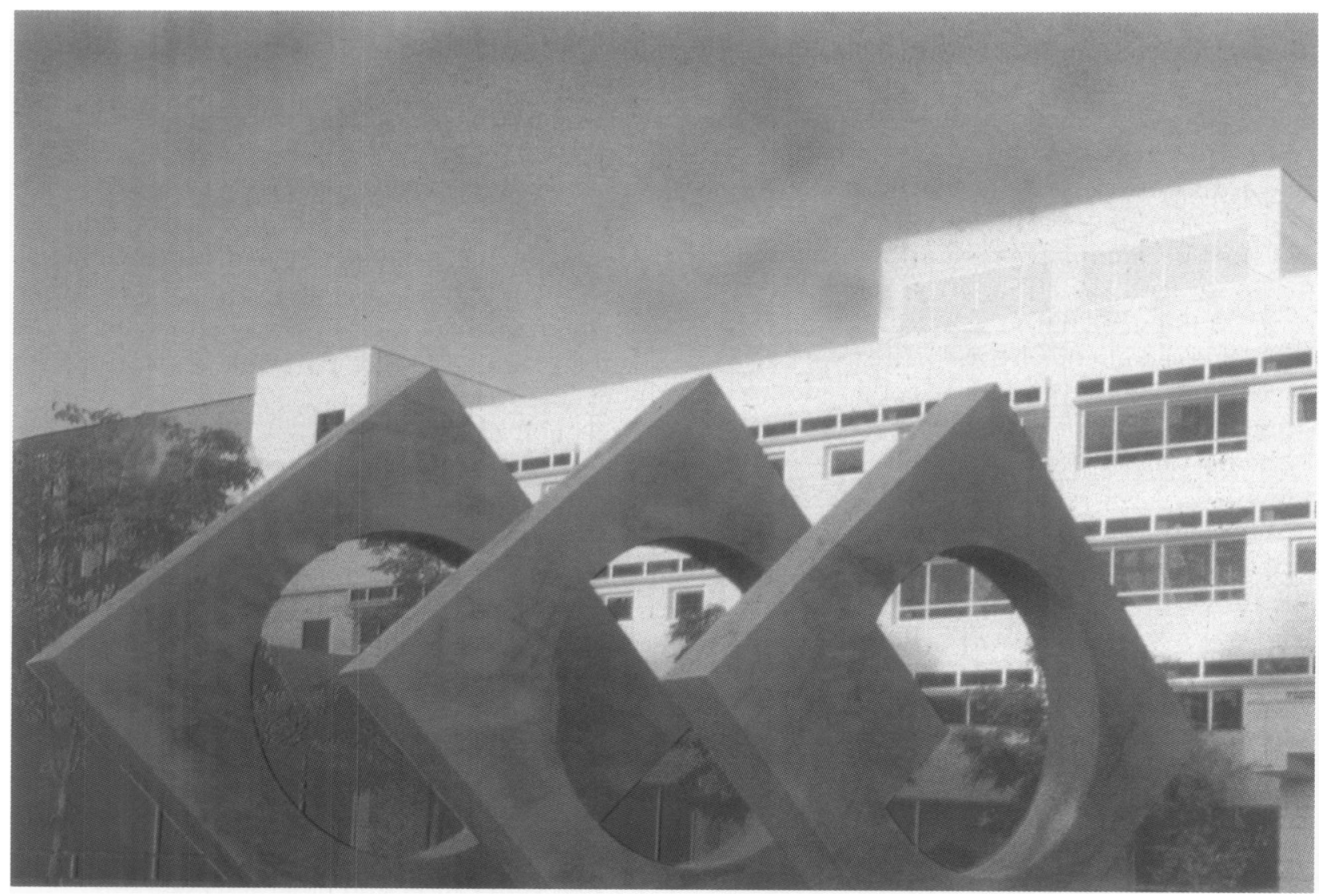

FIG. 1. Fox Chase Cancer Center

mechanisms of action and target molecules, researchers may be able to develop therapies to treat or reverse acquired immune deficiency syndrome by blocking the viral infection.

In other areas of virology, scientists are continuing their research on hepadnaviruses in an attempt to understand the molecular mechanisms of viral infection. This will enable them to design strategies that interfere with viral reproduction. Through study of the hepatitis delta agent, one scientist is searching for answers to this source of serious chronic liver disease and death in people chronically infected with hepatitis B.

Virologist Glenn Rall researches the dynamics of the immune response to viral infections of the central nervous system using neurotropic murine viruses, with emphasis on infection and function of the blood-brain barrier. He is also interested in the role of adhesion molecules in the regulation of lymphocyte traffic into the CNS.

\section{THE MOLECULAR ONCOLOGY PROGRAM}

Fox Chase's Molecular Oncology Program is led by Dr. Alfred G. Knudson Jr. A geneticist and a physician, Knudson is internationally recognized for his "two-hit" theory of cancer causation, which explains the relationship between the hereditary and nonhereditary forms of a cancer, and predicts the existence of anti-oncogenesgenes that can suppress cell growth. This nowconfirmed theory has advanced understanding of errors in the genetic program that turn normal cells into cancer cells. Knudson divides his time between Fox Chase and the National Cancer Institute $(\mathrm{NCI})$, where he was appointed as special adviser to Dr. Richard Klausner, Director of the NCI, while working with Dr. Joseph Fraumeni in NCI's new Division of Cancer Epidemiology and Genetics.

Fox Chase's molecular oncology researchers are integrating research from all the scientific 
divisions to study the molecular and genetic features of cancer in humans and animals, the basic molecular mechanisms of growth in the pathogenesis of cancer, and whether it is possible to reverse the process at the molecular level. Specific areas of study include genetic alteration in cancers, hereditary predisposition to cancer, oncogenes and tumor suppressor genes, transduction of growth signals, and control of the cell cycle. At the same time, the practical experience and observations of physicians treating cancer patients help formulate basic scientific questions. Insights gained from the program's approach, including clinical studies of the biology of human cancer, allow researchers to explore new ways to treat cancer patients or prevent cancer with genetic tools.

In some areas of special interest, Fox Chase scientists are involved in defining in molecular terms the cellular aspects of expression of the $p 53$ gene that account for its function as a tumor suppressor in diverse human tissues. Two fundamental areas of cancer biology-the mechanisms that a cell uses to avoid the killing properties of chemotherapeutic drugs and how cells transmit the internal signals that control basic activities such as growth-are another focus in molecular oncology.

A continued effort to identify genes involved in the pathogenesis of malignant mesothelioma has also been made at Fox Chase. In other work, investigators conducted a large-scale, multicenter study of alterations of the $A K T 2$ oncogene in ovarian and breast carcinomas. Amplification and overexpression of this gene were identified in nearly $15 \%$ of ovarian tumors but were rarely observed in breast cancers.

\section{THE HUMAN GENETICS PROGRAM AT FOX CHASE}

The world of genetics is a very different place than when Jack Schultz was the first staff member at the Institute for Cancer Research working on genetics. Schultz theorized the role of genetics in cancer with Drosophila (fruit flies). Rapid advances in the field of molecular genetics and genetic epidemiology have made the study of the genetic basis of common human diseases increasingly practical.

The combination of detailed genetic maps composed of easily characterized "markers" with powerful biomathematical methods has allowed the identification of genes responsible for many "single-gene" diseases. The unifying theme of molecular genetics research at Fox Chase is the application and extension of these methods to better understand the genetics of complex "multi-gene" diseases, which are responsible for most of the common human cancers, and to elucidate the interactions between genetic and environmental factors. This information can then be used to identify people at high risk for cancer and to devise potential strategies for prevention and treatment. Fox Chase continues to be a pioneer in the field of prevention research as the understanding of prevention's role becomes a more clear and necessary expansion of the future of medicine.

Under the leadership of Kenneth H. Buetow, Fox Chase's molecular genetics research is committed in a large part to the construction of human genetic maps and distribution of these maps to the scientific community. This effort is an essential component of the Human Genome Project, an international effort to map all human chromosomes and identify specific genes associated with cancer and other diseases.

To generate these maps, Dr. Buetow and his colleagues are developing new, semi-automated map construction procedures. Fox Chase is also responsible for providing computer-based tools that facilitate the storage, retrieval, and interpretation of the genetic map data as well as the primary data from which the maps are derived. For this unique effort, Dr. Buetow and his team received the Computerworld Smithsonian Award for science in 1995.

Some human geneticists are now researching the role of the metabolism of homocysteine, a key cellular chemical. High plasma levels of homocysteine place individuals at risk for a wide variety of developmental diseases. In addition, defects in homocysteine metabolism are frequently observed in human tumor cells. Fox Chase investigators in the human genetics working group are also interested in identifying, cloning, and characterizing genes involved in tumor progression, metastasis, and chemotherapeutic drug resistance.

One investigator is studying a hypothesis that a variation in the Cyp 19 gene can predict individual risk for breast cancer development. After analyzing Cyp19 in breast cancer patients and normal women, she found structural varia- 
tions between the two groups. She is further sequencing the "high-risk" and "low-risk" gene variants to identify their differences at the molecular level.

All three divisions at Fox Chase-basic science, medical science, and population scienceare involved in molecular medicine research to- ward the goal of reducing the burden of human cancer.

\section{ACKNOWLEDGMENT}

I thank Andrea Bozzelli for assistance with this article. 